\title{
Síndrome de choque tóxico por Streptococcus pyogenes en un hospital de la Ciudad de México
}

Toxic shock syndrome caused by Streptococcus in a hospital from the Mexico City

Síndrome de choque tóxico por Streptococcus pyogenes em um hospital da Cidade do México

Itzel Oralia Amaya Díaz de León, ${ }^{*}$ Marcelo Díaz Conde ${ }^{\ddagger}$

\section{RESUMEN}

El síndrome de choque tóxico por Streptococcus se describió por primera vez en 1978 por Todd y su equipo. Éste incluye datos de hipotensión, disoxia celular, aislamiento de Streptococcus pyogenes, así como la inclusión de dos o más de los siguientes criterios: síndrome de dificultad respiratoria aguda, insuficiencia renal, anormalidad hepática, coagulopatía y erupción cutánea con descamación. Es una enfermedad relativamente rara entre niños y adultos; representa una alta mortalidad, la cual oscila de 23 al $70 \%$. En más de la mitad de los casos, se desconoce la causa de entrada de este patógeno al torrente sanguíneo, aunque los pacientes con alcoholismo, en los extremos de la vida, con comorbilidades (como enfermedad renal crónica, insuficiencia hepática y leucemias) y que hayan sido sometidos a esplenectomía son más susceptibles al contagio y a infecciones severas. En los pacientes que desarrollan infecciones de tejidos blandos profundos, como fascitis necrosante o infección intrauterina, el dolor severo es el síntoma inicial más frecuente.

Palabras clave: Síndrome de choque tóxico, estreptococo, Streptococcus pyogenes, fascitis necrosante, inmunoglobulina humana.

\section{ABSTRACT}

Streptococcal toxic shock syndrome was first described by Todd and collaborators in 1978, this syndrome includes hypotension, traces of cellular dysoxia, isolation Streptococcus pyogenes; as well as two or more of the following criteria: acute respiratory distress syndrome, renal failure, liver abnormality, coagulopathy, skin rash with necrosis flaking of soft tissues. It is a relatively rare disease among children and adults, representing a high mortality ranging from 23 to $70 \%$. In more than half of the cases the cause of entry of this pathogen into the bloodstream is unknown, although patients with alcoholism, on the edge of death, with splenectomy, with comorbidities (chronic kidney disease, liver failure, leukemia) are more susceptible to infection, to acquire severe infections and shock. Cutaneous infections usually are followed by minor skin irritations (insect bites), $20 \%$ of the cases experience a flu-like syndrome (fever, chills, myalgia and diarrhea). In patients who develop deep soft tissue infections such as necrotizing fasciitis or intrauterine infection, the severe pain is the most common initial symptom of streptococcal TSS.

Keywords: Streptococcal toxic shock syndrome, Streptococcus, Streptococcus pyogenes, necrotizing fasciitis, human immunoglobulin.

\section{RESUMO}

A síndrome do choque tóxico estreptocócico foi descrita pela primeira vez por Todd et al em 1978, incluindo dados sobre hipotensão, disxia celular, isolamento de Streptococcus pyogenes, bem como 2 ou mais dos seguintes critérios: síndrome do desconforto respiratório agudo, insuficiência renal, anormalidade hepática, coagulopatia, erupção cutânea com descamação. É uma doença relativamente rara em crianças e adultos, representa uma alta mortalidade que varia de 23 a $70 \%$. Em mais da metade dos casos, a causa desse patógeno na corrente sanguínea é desconhecida, embora pacientes com alcoolismo, extremos da vida, com esplenectomia e comorbidades (como doença renal crônica, insuficiência hepática, leucemia) sejam mais suscetíveis à infecção e infecções graves. Em pacientes que desenvolvem infecções profundas dos tecidos moles, como fasceíte necrozante ou infecção intra-uterina, a dor intensa é o sintoma inicial mais frequente.

Palavras-chave: Síndrome de choque tóxico, estreptococo, Streptococcus pyogenes, faciite necrosante; imunoglobulina humana.

\section{* Unidad de Urgencias.}

₹ Unidad de Terapia Intensiva.

Hospital General «Dr. Manuel Gea González». Ciudad de México.

Recepción: 01/09/2019. Aceptación: 02/12/2019.

Este artículo puede ser consultado en versión completa en www.medigraphic.com/medicinacritica

\section{INTRODUCCIÓN}

La bacteria Streptococcus del grupo A, también conocido como Streptococcus pyogenes, es un patógeno responsable de una amplia variedad de enfermedades infecciosas. ${ }^{1,2}$ La estructura es un coco Gram-positivo no móvil, de catalasa negativa, no formador de esporas, anaerobio facultativo, producida en cadenas o parejas, y que se caracteriza por cocos ovoideos de 0.6-1 micrómetros de diámetro.

Su pared celular se compone de unidades repetidas de $\mathrm{N}$-acetilglucosamina y ácido $\mathrm{N}$-acetilmurámico -el peptidoglicano estándar-. Su patogenia depende de sus toxinas de tipo A, B y C. Estas toxinas actúan como superantígenos, que estimulan a las células $T$ mediante la unión de moléculas de MHC de clase II, resultando en la liberación de citoquinas masivas. ${ }^{3-5}$

El contacto de los pacientes con síndrome de choque tóxico (TSS, por sus siglas en inglés) puede desarrollar cualquiera de las siguientes infecciones: a) colonización asintomática, b) faringitis sintomática, c) bacteriemia sin estreptococos, d) fascitis necrosante, e) no colonización, f) TSS estreptocócico. La susceptibilidad del huésped a la infección está inversamente relacionada con la cantidad de anticuerpos contra factores de virulencia. ${ }^{6}$

Las infecciones cutáneas son más comunes en los niños de edad preescolar a finales del verano y principios del otoño en climas templados, siendo el mecanismo de propagación a través de gotas respiratorias o de contacto con los fómites. Las infecciones cutáneas suelen ser precedidas por irritaciones leves en la piel, tales como picaduras de insectos. ${ }^{4}$

EI TSS fue descrito por primera vez por Todd y su equipo en $1978 .^{2}$ Los criterios para el diagnóstico se enlistan en la Tabla 1.4-6

Las infecciones invasivas potencialmente mortales de $S$. pyogenes requieren de un reconocimiento precoz, diagnóstico definitivo y tratamiento agresivo temprano. ${ }^{7,8}$

Los síntomas iniciales del TSS estreptocócico dependen en gran medida del sitio de la infección primaria. Estas exotoxinas son altamente destructivas para la piel, el músculo y otros tejidos blandos, pudiendo causar: fiebre, choque y disfunción multiorgánica. ${ }^{1,6}$ EI TSS raramente se asocia con cultivos sanguíneos positivos, siendo los hemocultivos positivos hasta en $60 \% .^{2,5}$

Para ayudar al diagnóstico precoz de la infección de los tejidos blandos, se ha utilizado la exploración por 
tomografía computarizada, al igual que la resonancia magnética. ${ }^{3}$ Para el tratamiento, está el soporte hemodinámico, el uso temprano de antibióticos a base de penicilina; en caso de alergia eritromicina; o cuando se presenta datos de inestabilidad hemodinámica, clindamicina u antibióticos de amplio espectro. En caso de desarrollar fascitis necrosante o datos de síndrome compartimental, el tratamiento será quirúrgico de forma inmediata. ${ }^{4-6,9,10}$

A continuación, presentamos el caso de un hombre de 43 años de edad, con antecedente de epilepsia, quien desarrolló un síndrome de choque tóxico secundario a la infección por $S$. pyogenes. Dicho caso sirve como medio para generar una revisión en la literatura referente al tema, y, de esta manera, se pueda conocer los aspectos virulentos de dicho patógeno, inducir a la identificación de manera oportuna, así como incentivar la sospecha diagnóstica y proveer un tratamiento óptimo en fases tempranas.

\section{CASO CLÍNICO}

\section{Presentación del caso clínico}

Se trata de un paciente masculino de 43 años de edad, soltero, con antecedentes de epilepsia remanente de siete años desde su diagnóstico, con tratamiento basado en carbamazepina (200 mg cada 12 horas), sin un adecuado control médico ni supervisión en la toma regular de su tratamiento.

Su padecimiento lo inició dos días previos a su ingreso a la unidad de Urgencias, el cual se caracteriza por la presencia de dolor en el miembro pélvico derecho de moderada intensidad, con aumento de volumen y eritema que aumentaba a la deambulación, acompañado de cefalea holocraneana de intensidad moderada, astenia, adinamia e hiporexia.

Acudió con el médico facultativo, quien le proporcionó tratamiento basado en clindamicina (se desconoce la

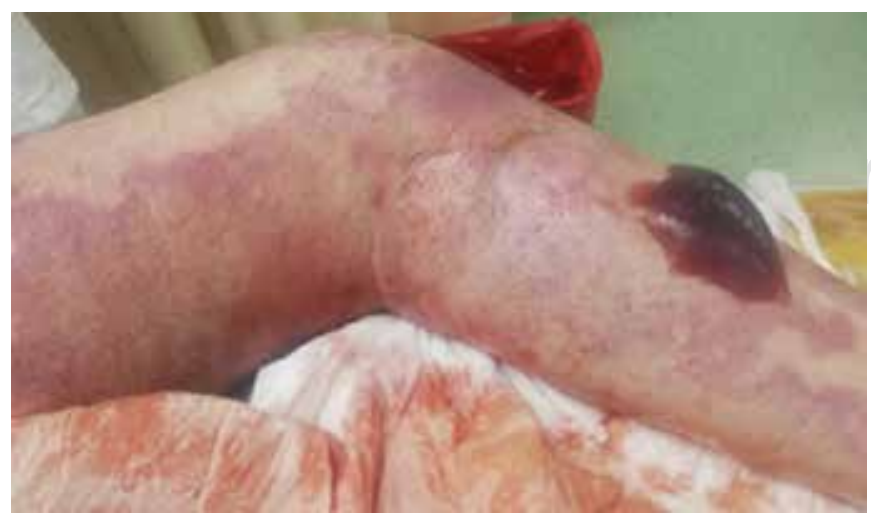

Figura 1: Se observa ampolla hemorrágica flácida en el tercio anterior de pierna derecha; resto: mancha purpúrica y edema.

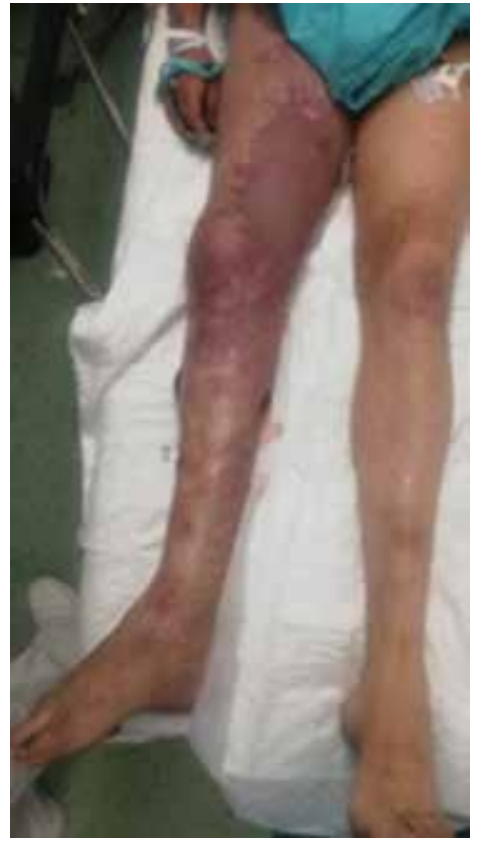

Figura 2:

Mancha de aspecto purpúrico, en tercio superior de muslo erosión en región proximal de muslo. dosis), y al no ver mejoría, se decidió acudir al Servicio de Urgencias del hospital referido en el presente caso.

A su llegada, se reportó como hipotenso y taquicárdico, con saturación al 87\%, presentando un índice de choque de 2.56, qSOFA de 2 puntos. A la exploración física, se observó la presencia de dermatosis localizada en extremidad inferior derecha, con extensión hacia el muslo y dorso del pie, caracterizada por una placa violácea, edematosa de bordes bien definidos, con algunas ampollas hemorrágicas de predominio en la pierna (Figuras 1 a 3).

Se decidió iniciar con el protocolo de atención para paciente séptico mediante reanimación a través de soluciones cristaloides, obtención de cultivos (hemocultivo y cultivo de flictenas), antibioticoterapia (ceftriaxona), analgesia (opioide débil) y tromboprofilaxis por alto riesgo de coagulación. Se solicitaron exámenes de laboratorios, cuyos resultados manifestaron: leucos: $\mathbf{8 . 3}$ 10^3/ $\mu \mathrm{L}$, neutros: $7.610^{\wedge} 3 / \mu \mathrm{L}$, hemoglobina: $15.4 \mathrm{~g} /$ dL, hematocrito: $47.1 \%$, plaquetas $17710^{\wedge} 3 / \mu \mathrm{L}$, bandas $31 \%$, creatinina: $4.72 \mathrm{mg} / \mathrm{dL}$, sodio: $129 \mathrm{mEq} / \mathrm{L}$, potasio: $3.8 \mathrm{mEq} / \mathrm{L}$, cloro: $92 \mathrm{mEq} / \mathrm{L}$, calcio: $7.8 \mathrm{mg} / \mathrm{dL}$, calcio corregido: $8.90 \mathrm{mg} / \mathrm{dL}$, fósforo: $6.84 \mathrm{mg} / \mathrm{dL}$, magnesio: $2.1 \mathrm{mg} / \mathrm{dL}$, bilirrubina total: $0.73 \mathrm{mg} / \mathrm{dL}$, bilirrubina directa: $0.39 \mathrm{mg} / \mathrm{dL}$, bilirrubina indirecta: $0.34 \mathrm{mg} /$ $\mathrm{dL}$, proteínas totales: $5.84 \mathrm{~g} / \mathrm{dL}$, albúmina: $2.62 \mathrm{~g} / \mathrm{dL}$, globulina: $3.22 \mathrm{~g} / \mathrm{dL}$, ALT $27 \mathrm{IU} / \mathrm{L}$, AST 51 IU/L, GGT 62 IU/L, FA 89 IU/L, DHL 210 IU/L, amilasa 33 U/L, lipasa $13 \mathrm{U} / \mathrm{L}$, CK $574 \mathrm{IU} / \mathrm{L}$, CKMB $30.6 \mathrm{ng} / \mathrm{mL}$, mioglobina $3,656 \mathrm{ng} / \mathrm{mL}$, troponina I 0.033 , TP $27.1 \mathrm{seg}$, INR 1.91, TTP $40.1 \mathrm{seg}$ y dímero D 3.62

El examen general de orina dio nitritos positivos, leucos en sedimentos 0-3 y bacterias moderadas; gaso- 


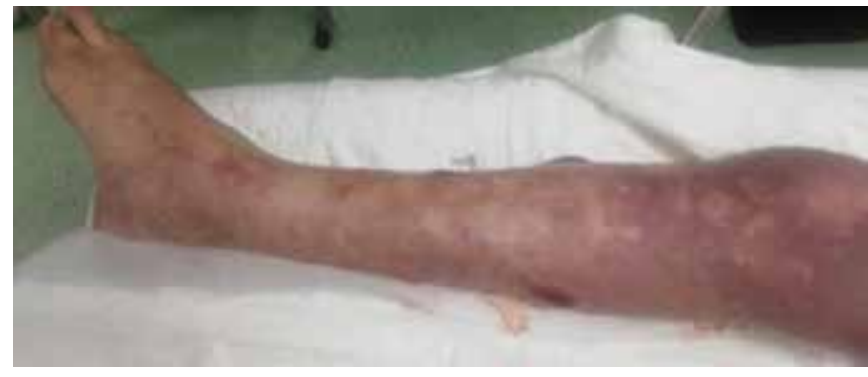

Figura 3: Cara anterior y lateral de pierna derecha con mancha violácea de bordes cartográficos y edema; además ampollas hemorrágicas flácidas.

metría arterial con acidosis metabólica con acidemia, con anión GAP amplio y acidosis láctica. Se tuvo la sospecha de trombosis venosa profunda, por lo que se realizó ultrasonido Doppler de miembro pélvico derecho, reportando la presencia de abundante edema de tejidos blandos en toda la extremidad, con trayectos de vena femoral común, femoral profunda, femoral superficial, poplítea y femorales posteriores con adecuada vascularidad, femoral anterior no valorable por abundante edema de tejidos blandos y flictenas, y sin datos de trombosis venosa profunda (Figura 4).

Se realizó manejo avanzado de la vía área por la presencia de dificultad respiratoria. Debido a que el paciente presentó poca respuesta a líquidos, se inició apoyo vasopresor. Se realizó ecocardiografía con equipo Philips $\mathrm{HD}$ con transductor sectorial, mostrando volumen de fin de diástole; $101 \mathrm{~mL}$, volumen de fin de sístole: 69 $\mathrm{mL}$, FEVI de $31 \%$, MAPSE $0.8 \mathrm{~cm}$, dilatación de cavidades izquierdas, hipocinesia generalizada del ventrículo izquierdo, con dilatación del mismo. Se observó imagen sugestiva de trombo móvil en ventrículo izquierdo de $4.9 \times 1.6 \mathrm{~cm}$, sin valvulopatías aparentes, vena cava inferior con diámetro de $1.31 \mathrm{~cm}$, colapsabilidad de $53 \%$, con el siguiente diagnóstico: disfunción sistólica moderada, con contractilidad disminuida longitudinal del $\mathrm{VI}$ e hipocinesia generalizada con trombo fresco intracavitario, sin valvulopatías aparentes y cavidades derechas normales (Figura 5).

Posteriormente se realizó cultivo de flictena de miembro pélvico derecho, donde se observó la presencia de Staphylococcus aureus (Figura 6) y el hemocultivo reportó Streptococcus pyogenes (Figura 6), por lo que se inició antibioticoterapia dirigida a base de cefalotina, esteroide para el manejo de choque refractario y tratamiento con inmunoglobulina. Desde su ingreso, el paciente presentó lesión renal aguda con rabdomiólisis CK > 5,629 IU/L y disminución de los flujos urinarios. El paciente presentó parada cardiaca, por lo que se inició reanimación cardiopulmonar durante 20 minutos, sin respuesta ni retorno a la circulación espontánea, por lo que se declaró clínicamente como fallecido.

\section{Comentarios}

Las infecciones graves por Streptococcus pyogenes ocurren esporádicamente con una tasa de ataque de sólo 2.7 a 3.6 por cada 100,000 habitantes en Europa, ${ }^{11}$ mientras que en Estados Unidos se reporta de 10,000 a 15,000 habitantes por año. ${ }^{12}$ Asimismo, se ha reportado síndrome de choque tóxico estreptocócico entre niños y adultos de todo el mundo, pero sigue siendo una enfermedad relativamente rara, con una tasa de mortalidad que oscila entre 23 al $70 \%$;, 13 por ello, el reconocimiento temprano de sus variadas presentaciones es esencial para un buen resultado. 3,10
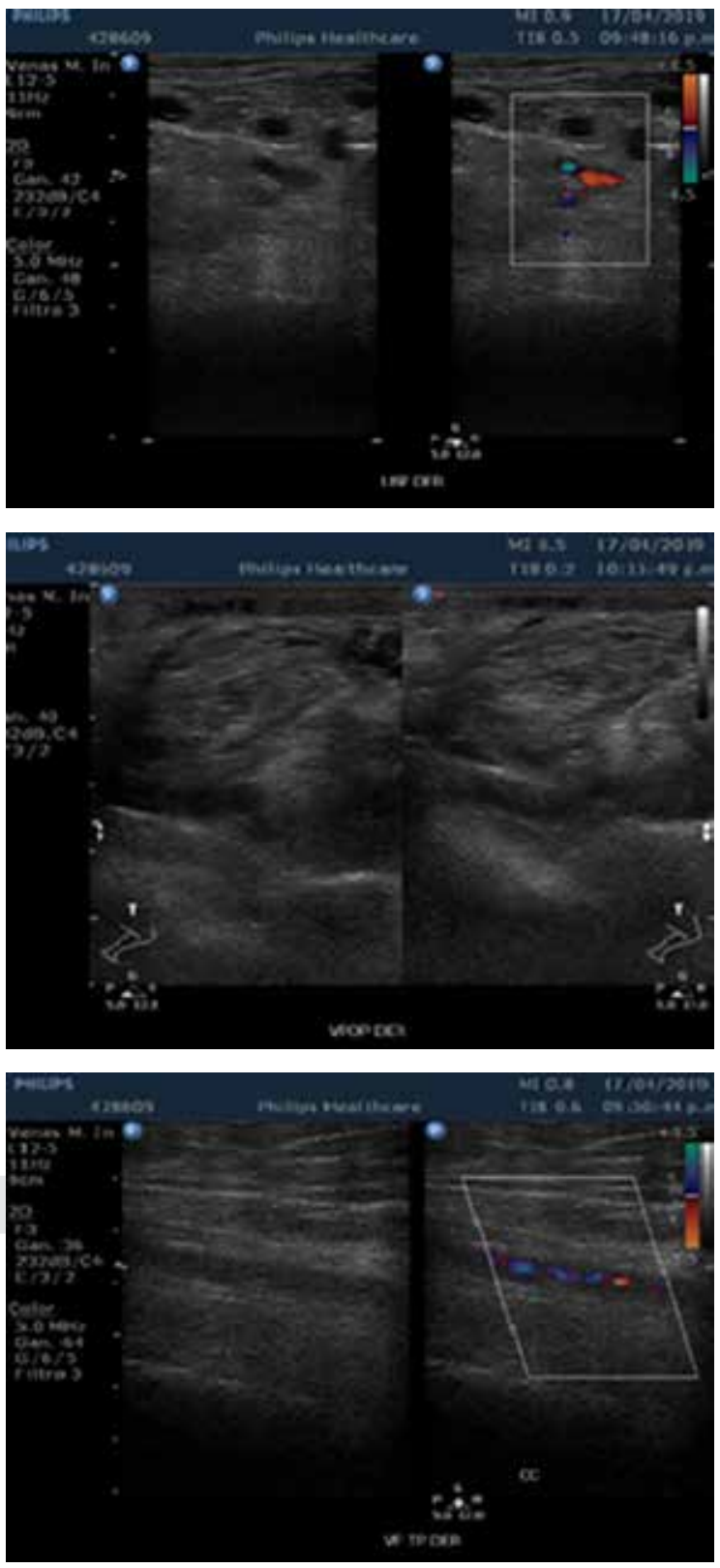

Figura 4: Ultrasonido Doppler de miembro pélvico afectado. 

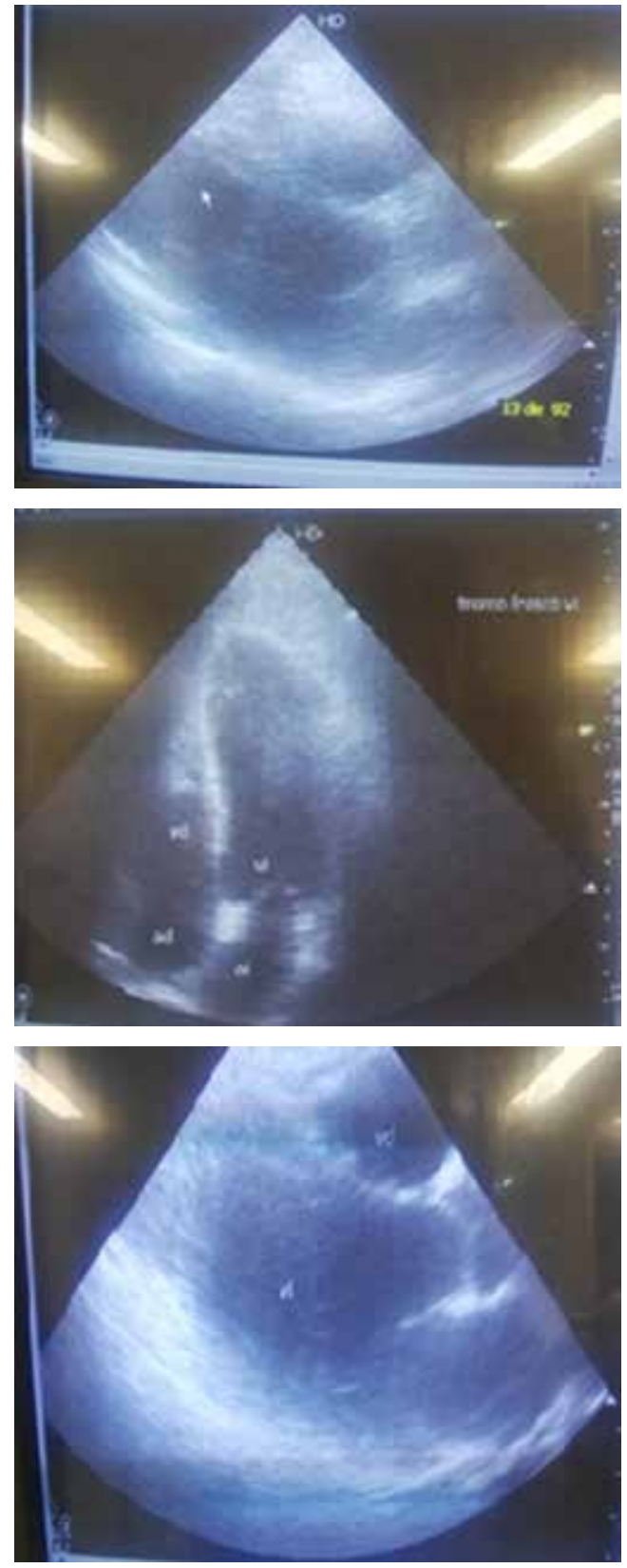

Figura 5: Ecocardiograma transtorácico.

Las exotoxinas estreptocócicas pirogénicas han sido denominadas como superantígenos, porque son capaces de superestimular el sistema inmunológico del huésped para producir una respuesta exagerada de la activación de células $T$ y la producción de citoquinas. ${ }^{3}$

Los estreptococos del grupo A producen una variedad de sustancias extracelulares, que tienen diferentes actividades antigénicas y biológicas. Éstos incluyen las citotoxinas, hemolíticas, ${ }^{5,7}$ estreptolisina $\mathrm{O}$ y S, así como la estreptoquinasa. Las exotoxinas pirogénicas estreptocócicas (SPE) de tipo A, B y C son las responsables de la fiebre y erupción de la escarlatina, y cada una tiene potentes propiedades tóxicas que alteran la defensa del huésped, aumentando la susceptibilidad al choque de la endotoxina, disminuyendo la respuesta de anticuerpos y permitiendo la inhibición de la función de macrófagos y la activación de linfocitos. ${ }^{1}$

Las exotoxinas pirogénicas estreptocócicas A y B inducen a las células mononucleares humanas a sintetizar no sólo el factor de necrosis tumoral- $\alpha$ (TNF- $\alpha$ ), sino también a la interleucina-1 $\beta$ (IL-1 $\beta$ ) y a la interleucina- 6 (IL-6), sugiriendo que el TNF podría mediar la fiebre, el choque y el tejido, lesión que es observada en pacientes con TSS estreptocócica. ${ }^{3}$

En cerca de $50 \%$ de los pacientes no se sabe el portal de virulencia. $^{9,14} \mathrm{~S}$. pyogenes provoca lesiones purulentas inflamatorias en el portal de entrada, a menudo en las vías respiratorias superiores o la piel. ${ }^{4}$ Aunque los pacientes alcohólicos, los pacientes en coma, los extremos de la vida, pacientes que han sido sometidos a esplenectomía y pacientes con enfermedades subyacentes (específicamente, enfermedad cardiaca crónica, pulmonar o renal, anemia de células falciformes, leucopenia, mieloma múltiple, cirrosis y diabetes), ${ }^{9}$ así como el uso de agentes antiinflamatorios no esteroideos pueden enmascarar los síntomas tempranos o predisponer al paciente a una infección estreptocócica más grave y a choque tóxico. . $^{2,5,7}$

La infección por S. pyogenes se puede presentar como una eritrodermia macular difusa, seguida de descamación y una afectación multisistémica, incluyendo afectación gastrointestinal, renal, hepática, respiratoria y del sistema nervioso central, cardiaco y disfunción hematológica, así como enfermedad de la gripe prodrómica

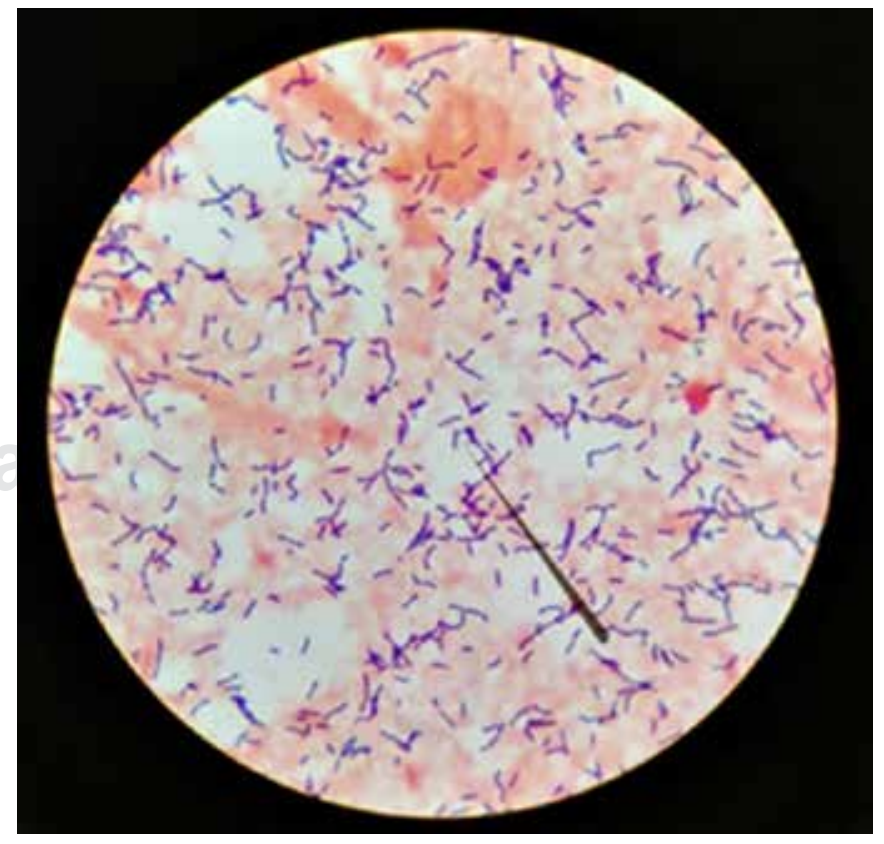

Figura 6: Microbiología: cocos Gram-positivos. 
Tabla 1: Criterios clínicos para síndrome de choque séptico por estreptococo basado en la definición de CDC.

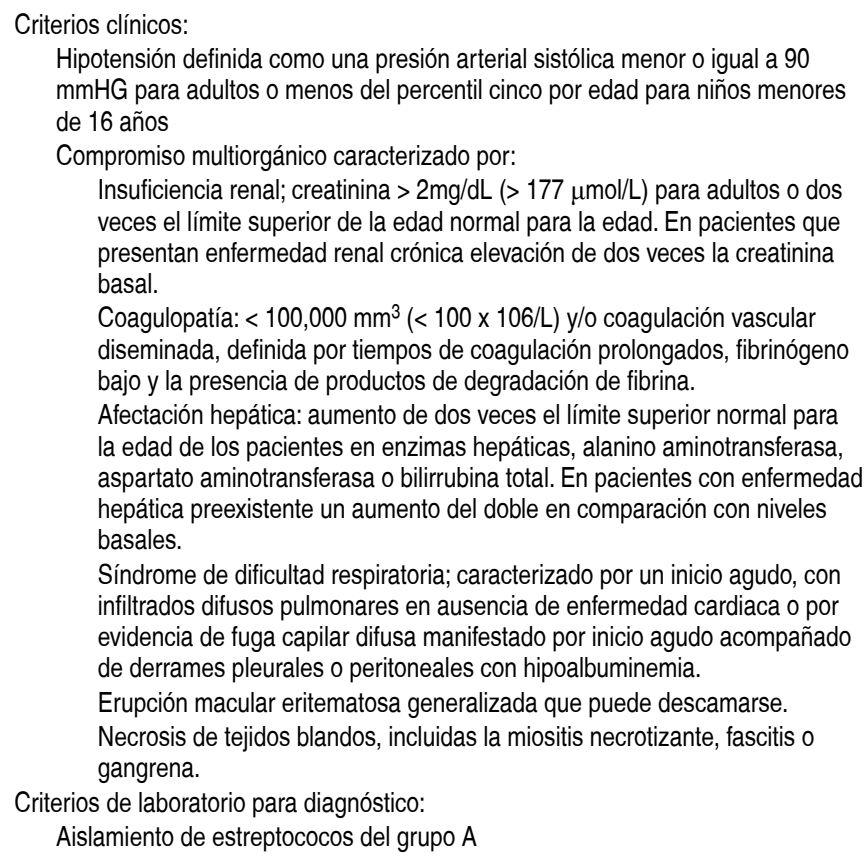
veces el límite superior de la edad normal para la edad. En pacientes que presentan enfermedad renal crónica elevación de dos veces la creatinina basal.

Coagulopatía: < 100,000 mm³ (< 100 x 106/L) y/o coagulación vascular diseminada, definida por tiempos de coagulación prolongados, fibrinógeno bajo y la presencia de productos de degradación de fibrina.

Afectación hepática: aumento de dos veces el límite superior normal para la edad de los pacientes en enzimas hepáticas, alanino aminotransferasa, aspartato aminotransferasa o bilirrubina total. En pacientes con enfermedad hepática preexistente un aumento del doble en comparación con niveles basales.

Síndrome de dificultad respiratoria; caracterizado por un inicio agudo, con infiltrados difusos pulmonares en ausencia de enfermedad cardiaca o por evidencia de fuga capilar difusa manifestado por inicio agudo acompañado de derrames pleurales o peritoneales con hipoalbuminemia.

Erupción macular eritematosa generalizada que puede descamarse.

Necrosis de tejidos blandos, incluidas la miositis necrotizante, fascitis 0 gangrena.

Criterios de laboratorio para diagnóstico:

Aislamiento de estreptococos del grupo $\mathrm{A}$

*Las manifestaciones clínicas no necesitan ser detectadas dentro de las primeras $48 \mathrm{~h}$ de hospitalización o enfermedad.

(visto en 20\%), dolor en una extremidad (el síntoma inicial más común), hinchazón localizada y eritema (el signo de presentación más frecuente), y vesículas y bullas, que progresan hasta miositis o fascitis necrosante..$^{2,5,14}$

La fiebre es el signo de presentación más frecuente del TSS estreptocócico. ${ }^{2}$ La confusión está presente en $55 \%$ de los pacientes y el deterioro neurológico que progresa al coma. La hinchazón localizada progresa rápidamente en el sitio de la fascitis necrosante y la miositis; la afectación renal es relativamente común, evidenciada por la presencia de hemoglobinuria y valores de creatinina sérica, los cuales estuvieron en promedio 2.5 veces por arriba de lo normal.

En la evaluación de pacientes con celulitis o infección de la herida que parezca tóxica, la creatina quinasa sérica (CK) y los niveles de proteína C-reactiva pueden ser notablemente elevados en aquéllos con fascitis necrosante o miositis. En $90 \%$ de los pacientes con TSS estreptocócico, el síndrome de distrés respiratorio agudo es lo suficientemente grave como para requerir oxígeno suplementario, intubación y ventilación mecánica. Invariablemente, dentro de las 24 horas el paciente experimenta fiebre, hipotensión, hinchazón masiva de la zona afectada y evidencia de celulitis. ${ }^{6}$

La fascitis necrosante es una infección subcutánea que destruye la grasa y la fascia subyacente, la cual progresa muy rápidamente, teniendo una alta mortalidad. ${ }^{1}$ Dentro de las 24 horas de la lesión inicial, hay un desarrollo agresivo de la hinchazón, calor, eritema e hipersensibilidad con una rápida propagación. Durante las próximas 24 a 48 horas, el eritema se oscurece, cambiando de rojo a púrpura, y posteriormente, a azul, formándose ampollas y bullas que contienen fluido amarillo claro. En el cuarto o quinto día, las áreas púrpuras se vuelven francamente gangrenosas. Desde el séptimo hasta el décimo día, la línea de demarcación se define fuertemente, y la piel muerta comienza a separarse en los márgenes, revelando una extensa necrosis del tejido subcutáneo. 2,5,6

La miositis estreptocócica se caracteriza por un dolor severo, hinchazón y eritema, pudiendo ser los únicos hallazgos físicos, ${ }^{2,3}$ cuya tasa de letalidad oscila de 80 al $100 \% .^{3}$ El examen patológico muestra infiltrados purulentos reemplazados por necróticos, tal vez músculo-hemorrágico y fascia. ${ }^{3}$

En ciertos estudios de laboratorio, microbiología e imágenes, el recuento de glóbulos blancos puede ser normal o sólo moderadamente elevados en el momento de la admisión, generalmente se acompañan de una marcada elevación de los neutrófilos inmaduros circulantes, ${ }^{5,6}$ anemia hemolítica, elevación de creatinina trombocitopenia, aumento de los tiempos de coagulación, hipertransaminasemia e hipoxemia en caso de lesión pulmonar aguda. ${ }^{2,5,9}$

\section{Diagnóstico diferencial}

Los diagnósticos erróneos iniciales clásicos son trombosis venosa profunda, isquemia de las extremidades, gastroenteritis, peritonitis, síndrome coronario agudo, pericarditis y meningitis. ${ }^{5}$

\section{Tratamiento antibiótico}

La penicilina sigue siendo el fármaco de elección para el $S$. pyogenes. Si se produce alergia a la penicilina,,$^{1,3}$ un fármaco alternativo es eritromicina y clindamicina. $.4,6,9,13$ La resistencia a la clindamicina y eritromicina ha ido en aumento, la cual puede estar presente hasta en 15 a $20 \%$ de los pacientes. Las alternativas a éstas incluyen vancomicina y cefalosporinas de primera, segunda o tercera generación..$^{9,11}$

Además de los antibióticos, el paciente con miositis por $S$. pyogenes o fascitis necrosante requiere desbridamiento quirúrgico. .-6 $^{-}$

El síndrome de choque tóxico estreptocócico tiene un curso rápido y con frecuencia desenlace fatal, ${ }^{1}$ por lo que el tratamiento quirúrgico se recomienda para la eliminación de la fuente de infección. ${ }^{10}$ El desbridamiento quirúrgico o drenaje es a menudo esencial para reducir la carga bacteriana. ${ }^{3}$

Las inmunoglobulinas inician en enfermedades invasivas de GAS (estreptococo del grupo A) que no responde a tratamiento antibiótico ${ }^{7,13,14}$ su beneficio es 
difícil de probar porque el número limitado de casos excluye los resultados de una potencia estadística suficiente. ${ }^{7}$ Las dosis variaron de 0.4 a $1 \mathrm{~g} / \mathrm{kg}$, y el número de dosis administradas difiere. Los pacientes reciben la primera dosis de IVIG dentro de las primeras 24 a 48 horas después del diagnóstico. Los pacientes con más fallas orgánicas deberían de recibir mayor dosis de $2 \mathrm{~g} /$ $\mathrm{kg}$ y más días de medicación. ${ }^{13}$

La utilidad de ECMO (oxigenación por membrana extracorpórea) en adultos con sepsis sigue siendo controversial, aunque se está investigando como terapéutica valiosa para los pacientes con disfunción cardiovascular refractaria, especialmente cuando se introdujo inmediatamente después de desarrollar choque séptico. ${ }^{8}$

El síndrome de choque séptico por estreptococo es una forma de presentación por infección de estreptococo poco común con una alta mortalidad si no se detecta a tiempo y se da un tratamiento oportuno, por el que se desconozca su vía de entrada, llevando al paciente a una disfunción multiorgánica en cuestión de horas. La base del tratamiento sigue siendo el uso de antibiótico a base de penicilina y, en estados de gravedad, clindamicina, además de tratamiento quirúrgico.

Sigue estando en debate el uso de ECMO e inmunoglobulina, ya que el reporte de éxito de éstos es limitado y la mayoría se encuentra en reportes de caso clínico. Haber detectado un caso dentro de nuestro hospital e identificarlo, nos ayudará a estar más alerta para el diagnóstico temprano y prevenir complicaciones fatales que disminuyan la probabilidad de fallecimiento.

\section{BIBLIOGRAFÍA}

1. Wood TF, Potter MA, Jonasson O. Streptococcal toxic shock-like syndrome. The importance of surgical intervention. Ann Surg. 1993;217(2):109-114. doi: 10.1097/00000658-19930200000003.

2. Stevens DL. Streptococcal toxic-shock syndrome: spectrum of disease, pathogenesis, and new concepts in treatment. Emerg Infect Dis. 1995;1(3):69-78.
3. Fox KL, Born MW, Cohen MA. Fulminant infection and toxic shock syndrome caused by Streptococcus pyogenes. J Emerg Med. 2002;22(4):357-366.

4. Patterson MJ. Streptococcus. In: Baron S, editor. Medical microbiology. 4th edition. Galveston (TX): University of Texas Medical Branch at Galveston; 1996. Chapter 13. Available from: https://www.ncbi.nlm.nih.gov/books/NBK7611/

5. Schmitz M, Roux X, Huttner B, Pugin J. Streptococcal toxic shock syndrome in the intensive care unit. Ann Intensive Care. 2018;8(1):88.

6. Stevens DL. Streptococcal toxic shock syndrome associated with necrotizing fasciitis. Annu Rev Med. 2000;51:271-288.

7. Baxter M, Morgan M. Streptococcal toxic shock syndrome caused by group g streptococcus, United Kingdom. Emerg Infect Dis. 2017;23(1):127-129.

8. Mizuguchi Y, Taniguchi N, Takahashi A. Successful treatment of out-of-hospital cardiopulmonary arrest due to streptococcal toxic shock syndrome - effectiveness of extracorporeal membrane oxygenation and the rapid antigen group $A$ streptococcus test: a case report. J Med Case Rep. 2018;12(1):244.

9. Reich HL, Crawford GH, Pelle MT, James WD. Group B streptococcal toxic shock-like syndrome. Arch Dermatol. 2004;140(2):163-166.

10. Wilkins AL, Steer AC, Smeesters PR, Curtis N. Toxic shock syndrome - the seven Rs of management and treatment. $J$ Infect. 2017;74 Suppl 1:S147-S152.

11. Lamagni TL, Efstratiou A, Vuopio-Varkila J, Jasir A, Schalén C; Strep-EURO. The epidemiology of severe Streptococcus pyogenes associated disease in Europe. Euro Surveill. 2005;10(9):179-184.

12. Centers for Disease Control and Prevention (CDC). Invasive group A streptococcal infections--United Kingdom, 1994. MMWR Morb Mortal Wkly Rep. 1994;43(21):401-402.

13. Shah PJ, Vakil N, Kabakov A. Role of intravenous immune globulin in streptococcal toxic shock syndrome and Clostridium difficile infection. Am J Health Syst Pharm. 2015;72(12):1013-1019.

14. Georgouras KE, Konya J, Pang B. Toxic streptococcal syndrome. In: Stanford DG. The Dertmatology Centre. Sydney, New South Wales, Australia: Liverpool Hospital; 1997.

\section{Correspondencia:}

Dr. Marcelo Díaz Conde

Calzada de Tlalpan Núm. 4800,

Sección XVI, Alcaldía Tlalpan,

14080, Ciudad de México, México.

Teléfono: 4000300

E-mail: marcelomdc270681@ hotmail.com 